\title{
OPHTHALMIC IN-SITU SUSTAINED GEL OF CIPROFLOXACIN, PREPARATION AND EVALUATION STUDY
}

\author{
FADIA YASSIR AL-BAZZAZ ${ }^{1 *}$, MYASAR AL-KOTAJI ${ }^{2}$ \\ 1,2Department of Pharmaceutics, College of Pharmacy, University of Mosul, Mosul, Iraq \\ Email: fadiayassir@gmail.com
}

Received: 23 Apr 2018, Revised and Accepted: 06 Jun 2018

\begin{abstract}
Objective: This work aims to formulate and evaluate an ophthalmic in-situ gel of ciprofloxacin hydrochloride (HCl) using poloxamer 407 (P407) as a gelling agent and hydroxypropyl methylcellulose (HPMC) as a viscosity modifier. The objective of this work was to prolong the contact time as the in-situ gel will be converted into a gel upon contact with the cul-de-sac.

Methods: Ciprofloxacin $\mathrm{HCl}$ ophthalmic in-situ gel was prepared by utilizing (P407) as a temperature-dependent polymer while hydroxypropyl methylcellulose was used as a viscosity modifier. The system was evaluated for physical appearance, $\mathrm{pH}$, drug content, sterility, irritancy and stability. In addition, gelation temperature and a viscosity at different shear rates and different temperatures were studied. The compatibility of the polymer with ciprofloxacin was studied by using fourier transform infrared spectroscopy (FTIR). The in vitro release of the drug was also evaluated and supported by a preliminary in vivo test.

Results: The results showed that the prepared formulas were clear, with acceptable pH and the drug contents were within the acceptable limits. FTIR results detected no incompatibility between poloxamer 407 and ciprofloxacin HCl. Notably, the viscosity of the system showed a pseudoplastic behaviour where a reduction in viscosity upon increasing the shear rate was observed. The in vitro release study confirmed the prolongation of the release of the optimized formula (F6) up to $8 \mathrm{~h}$. Upon application of F6 into eyes of rabbits there was no irritancy. In addition, in vivo elimination study showed a prolonged contact for the in-situ gel in comparison with the rapid clearance of eye drop. Stability study indicated the stability of the optimized formula (F6).
\end{abstract}

Conclusion: The prepared optimized formula (F6) represents a successful, safe, stable and prolonged release in-situ gel formula of ciprofloxacin.

Keywords: Ophthalmic in-situ gel, Poloxamer, Ciprofloxacin $\mathrm{HCl}$, Solution-gel transition

(C) 2018 The Authors. Published by Innovare Academic Sciences Pvt Ltd. This is an open access article under the CC BY license (http://creativecommons.org/licenses/by/4.0/) DOI: http://dx.doi.org/10.22159/ijap.2018v10i4.26885

\section{INTRODUCTION}

The most common and well-accepted route of administration for the treatment of many eye conditions is the topical application of drugs to the eye. Blinking, baseline and reflex lachrymation, and drainage remove foreign ingredients, including drugs, from the surface of the eye. Furthermore, the physiology, anatomy and barrier role of the cornea decreases the rapid absorption of drugs [1]. The exceptional anatomical and physiological nature and protective mechanisms of the eye make the targeting of drugs to eye tissues one of the most extreme challenges in drug delivery [2]. A substantial task for the formulator is to avoid the defensive barriers of the eye without causing permanent tissue impairment [3].

Formulation of ophthalmic drug delivery systems has many challenges, but also offers many prospects to overcome the insufficiencies of the present formulations. As a result of the restricted area and time for absorption in the eye, the residence time of most conventional ocular solutions is 5-25 min and only 1-10\% of the topically applied drug is absorbed [2]. In addition to the various protective mechanisms, the corneal epithelium has a compound hydro-and lipophilic character that limits drug absorption. The conjunctiva plays an important role in drug elimination from the ocular surface due to its rich blood flow, large surface area and more permeable membrane compared to the corneal membrane. There is consequently a need for the recurrent instillation of eye drops, which is accompanied by distress and a reduction in patient compliance, especially in long-term therapy [4].

Therefore, an increase in the contact time between dosage form and the corneal surface is required to overcome these difficulties. This can be achieved by gelling systems. In-situ gelling systems can be defined as viscous liquids, which undergo a phase transition from solution to gel when applied to the human body. The triggers to phase change are either temperature, $\mathrm{pH}$ or ionic strength. In-situ gelling systems permit precise and reproducible administration of a drug not like preformed gels and are capable of extending the formulation's residence time to the mucosal surface due to postinstillation gelling [5].

The most common polymers used in the formulation of in-situ gel include poloxamer, carbopol and alginates polymers for temperature-dependent, pH-dependent, and ionic-dependent systems respectively.

Poloxamer 407 (P407) or what is known commercially as Pluronic ${ }^{\circledR}$ F-127 consists of about $70 \%$ ethylene oxide, which accounts for its hydrophilicity. P407 is a 12,000-Da copolymer; it is non-toxic, liquid at $4{ }^{\circ} \mathrm{C}$ and converts to a semisolid gel at body temperature [6]. P407 is common in ophthalmic use, where it is changed into a colorless, optically clear and transparent gel at temperatures above $35^{\circ} \mathrm{C}$ [2]. P407 molecules aggregate into micelles when the temperature rises. This micellization is the very first step in the gelling process which occurs owing to the dehydration of hydrophobic propylene oxide blocks. The dehydrated polyoxypropylene form the core of the spherical micelles while the outer shell contains hydrated swollen polyoxyethylene chains. Then as temperature increases further, gelation occurs for sufficiently concentrated samples [7].

Ciprofloxacin hydrochloride (HCL) (fig. 1) is a potent broadspectrum antibiotic of fluoroquinolones, the in vitro antibacterial activity is greater than other antibiotics. Additionally, ciprofloxacin has an effective topical antibiotic activity with a low frequency of spontaneous resistance, therefore, it is a favorable drug for the treatment of bacterial keratitis [8].

This work aims to formulate and evaluate a thermo-sensitive ocular insitu gel of ciprofloxacin $\mathrm{HCl}$ using poloxamer 407 (P407) as a gelling agent and hydroxypropyl methylcellulose (HPMC) as a viscosity modifier. Visual appearance, clarity, $\mathrm{pH}$ and sol-gel transition temperature of formulas were tested. Comprehensive rheological properties of the prepared formula were also studied. In addition, 
irritancy test of the best formula was assessed. The release of the drug was studied by using in vitro dissolution test. In order to study the retention of the gel in the eye, a pioneer work was conducted using an auto refractometer apparatus to track the contact time of ciprofloxacin gel in comparison to marketed eye drop of ciprofloxacin. In addition, sterility test and stability test were also conducted.<smiles>O=C(O)c1cn(C2CC2)c2cc(N3CCNCC3)c(F)cc2c1=O</smiles>

Fig. 1: The chemical structure of ciprofloxacin hydrochloride [9]

\section{MATERIALS AND METHODS}

\section{Materials}

Ciprofloxacin hydrochloride was kindly supplied by Pioneer company for pharmaceutical industry (Sulaymania, Iraq), Pluronic ${ }^{\circledR F}$-127 (P407) (Sigma Aldrich CO. USA), hydroxypropyl methylcellulose (HPMC) (HIMEDIA), sodium chloride (Akzonobel, Denmark), sodium bicarbonate (Solvay, Germany), Calcium chloride dihydrate (VIGROUS Group, China), Sodium acetate (Merck, Germany), Fluorescein sodium ophthalmic strips U. S. P. (OptiGlo, India). All other chemicals were of analytical grade.

\section{Preparation of the in-situ gels}

Twelve different formulas of ciprofloxacin $\mathrm{HCl}$ in-situ gel were prepared by using different concentrations of (P407) with different concentrations of HPMC (table 1).

An in-situ gel of ciprofloxacin was prepared by using the cold method [10]. The required amount of (P407) was added to acetate buffer ( $\mathrm{pH} \mathrm{4.5)} \mathrm{(at} 4^{\circ} \mathrm{C}$ ) with gentle mixing and then allowed to hydrate overnight at the same temperature. The required amount of viscosity enhancing agent, HPMC, was dissolved in hot acetate buffer and cooled to $4{ }^{\circ} \mathrm{C}$ and then added to polymer solutions. Ciprofloxacin $\mathrm{HCl}$ was dissolved in the required amount of acetate buffer and then sodium chloride and benzalkonium chloride were added to the solution. After that, the drug solution was added to the polymer solution and mixed.

The samples were then transferred to vials and stored in a refrigerator overnight. Finally, the vials were sterilized by autoclave at $121^{\circ} \mathrm{C}$ at $15 \mathrm{psi}$ for $20 \mathrm{~min}$.

Table 1: Composition of ophthalmic in-situ gels of ciprofloxacin hydrochloride $\%(w / w)$

\begin{tabular}{|c|c|c|c|c|c|}
\hline Formulas & Ciprofloxacin HCl & (P407) & HPMC & Benzalkonium chloride & Sodium chloride equivalent to \\
\hline F1 & 0.3 & 15 & 0.2 & 0.02 & 0.9 \\
\hline F2 & 0.3 & 15 & 0.4 & 0.02 & 0.9 \\
\hline F3 & 0.3 & 15 & 0.6 & 0.20 & 0.9 \\
\hline $\mathrm{F} 4$ & 0.3 & 16.25 & 0.2 & 0.02 & 0.9 \\
\hline F5 & 0.3 & 16.25 & 0.4 & 0.02 & 0.9 \\
\hline F6 & 0.3 & 16.25 & 0.6 & 0.02 & 0.9 \\
\hline F7 & 0.3 & 17.5 & 0.2 & 0.02 & 0.9 \\
\hline F8 & 0.3 & 17.5 & 0.4 & 0.02 & 0.9 \\
\hline F9 & 0.3 & 17.5 & 0.6 & 0.02 & 0.9 \\
\hline F10 & 0.3 & 20 & 0.4 & 0.02 & 0.9 \\
\hline F11 & 0.3 & 16.25 & 0 & 0.02 & 0.9 \\
\hline $\mathrm{F} 12$ & 0.3 & 17.5 & 0 & 0.02 & 0.9 \\
\hline
\end{tabular}

\section{Evaluation of the in-situ gel}

\section{Clarity and pH}

All prepared formulas were evaluated for clarity by visual observation against a black and white background [11]. pH was measured using digital pH meter (Mettler Toledo/Germany).

\section{Drug content}

The drug content was determined by taking $0.2 \mathrm{ml}$ of the formula and completing the volume to $100 \mathrm{ml}$ with acetate buffer $\mathrm{pH} 4.5$. Ciprofloxacin concentration was then detected by measuring the absorbance at $278 \mathrm{~nm}$ by using UV-Vis spectrophotometer (Specord 50 , analytic Jena/Germany).

\section{Fourier transform infrared spectroscopy (FTIR)}

FTIR spectrometer (Thermo Scientific, USA) was used to record the FT-IR spectra of ciprofloxacin, P407 and their physical mixture. Sufficient amount (2-4 mg) of the sample was placed to form a thin film covering the diamond window.

\section{Measurement of the solution-gel transition temperature}

Tube tilting method was used to measure gelation temperature [12]. Two millilitres of the refrigerated formula were transferred to a test tube. The tube was maintained in a thermostatically controlled digital water bath (memmert, Germany) at $4{ }^{\circ} \mathrm{C}$. The temperature of the water bath was increased gradually in increments of $3{ }^{\circ} \mathrm{C}$ at the beginning of the experiment and then $1{ }^{\circ} \mathrm{C}$ increments in the region of sol-gel transition temperature $\left(25-34{ }^{\circ} \mathrm{C}\right)$ and $0.1{ }^{\circ} \mathrm{C}$ when it approaches gelation. The gelation was considered to occur when the meniscus of the formula would no longer move upon tilting through a $90^{\circ}$ angle [13].

\section{Gelling capacity}

The gelling capacity was determined by placing one drop of the formula in a test tube containing $2 \mathrm{ml}$ of freshly prepared simulated tear fluid (STF) and equilibrated at $37^{\circ} \mathrm{C}$ and visually assessing the formation of a gel, noting the time for gelation and the time taken for the formed gel to be dissolved [14]. STF composed of NaCl-0.67 g, $\mathrm{NaHCO}_{3}-0.20 \mathrm{~g}, \mathrm{CaCl}_{2} 2 \mathrm{H}_{2} \mathrm{O}-0.008 \mathrm{~g}$ and distilled water to $100 \mathrm{~g}$ [15].

\section{Rheological studies}

Rheological properties of the prepared ciprofloxacin $\mathrm{HCl}$ in-situ gels were measured using viscometer EZ-lock spindle coupling helipath stand (Brookfield Engineering Laboratories Inc., Model DV2T, USA) connected to a digital thermostatically controlled circulating water bath with spindle SC4-18. The viscosity was determined for each sample at a different shear rate and temperature. The angular velocity increased gradually from 0.1 to $50 \mathrm{rpm}$ while the temperature increased from 22 to $36^{\circ} \mathrm{C}$.

\section{Sterility test}

Sterility test was intended for identifying the presence of viable microorganisms and performed for aerobic/anaerobic bacteria and fungi by using fluid thioglycolate medium and soybean-casein digest medium. Two $\mathrm{ml}$ of the formula were withdrawn with a sterile syringe then aseptically transferred to thioglycolate medium and soybeancasein digest medium separately. The inoculated media were incubated for $14 \mathrm{~d}$ at $30-35{ }^{\circ} \mathrm{C}$ in case of fluid thioglycolate medium and at $20-25^{\circ} \mathrm{C}$ in case of soybean-casein digest medium [16].

\section{In vitro drug release study}

A modified dissolution test was used for evaluating the in vitro release profile [17]. A glass cylinder $(2.3 \times 8 \mathrm{~cm}$ in dimensions $)$, open at both 
ends, was used for the purpose of the study. Whatman ${ }^{\circledR}$ membrane previously soaked overnight in STF was taken and tied to one end of the cylinder by using parafilm and an elastic ring. One $\mathrm{ml}$ of the formula was accurately added on the membrane. Further, the glass cylinder was fixed to the shaft of USP apparatus I, in place of the basket. The cylinder was then suspended in the release medium (200 $\mathrm{ml}$ ) maintained at $37 \pm 0.5^{\circ} \mathrm{C}$ in a way that the membrane just touched the release medium. The speed of the device shaft was set at $50 \mathrm{rpm}$. Aliquots were withdrawn at different time intervals of $0.5,1,2,3,4,6$, 8 and $10 \mathrm{~h}$ and compensated by equal volumes of dissolution medium after each withdrawal. Aliquots were filtered and then analyzed by UV spectrophotometer at $272 \mathrm{~nm}$. The cumulative percentage of drug released was calculated and plotted against time.

\section{Ocular irritancy test}

Ocular irritation studies were conducted on 3 healthy female Newzeland albino rabbits weighing $1.5-2 \mathrm{~kg}$. They are obtained from the animal's house in veterinary college/university of Mosul (animal studies were approved by the postgraduate specialist committee in the college of pharmacy/university of Mosul, approval no. 12/2017-1). The animals, housed in an air-conditioned and in a light-controlled room at $20 \pm 1{ }^{\circ} \mathrm{C}$ and $50 \pm 5 \% \mathrm{R}$. H., were given a standard diet and water. The modified Draize technique [18] was designed for measuring the ocular irritation potential of the ophthalmic product. One drop of optimized in-situ gel formula was placed in the lower cul-de-sac of the left eye while the right eye left untreated and considered as a negative control. The rabbits' eyes were tested periodically after a different time interval of $1 \mathrm{~h}, 2 \mathrm{~h}, 24 \mathrm{~h}, 48$ $\mathrm{h}$, and $72 \mathrm{~h}$ after administration of treatment. Both eyes were checked by an ophthalmologist for redness, swelling and watering.

\section{In vivo fluorescein tracking study}

To reach the goal of studying the elimination time of the in-situ gels, fluorescein sodium salt was dispersed in the marketed eye drop and F6 by using fluorescein sodium ophthalmic strips U. S. P. (OptiGlo, India). One drop of each preparation was added into the lower conjunctival sac of rabbit $(\mathrm{n}=3)$, an autorefractometer (Huvitz, Korea) with blue light was used to monitor the disappearance of the fluorescein sodium salt. At selected time intervals the eyes were examined, when only a minute amount or none of the gel remained it was considered as lost from the eye. The preceding time for the check was defined as the elimination time.

\section{Stability study}

The stability of the optimized in-situ gel was conducted on the selected formula (F6). Ten ml of (F6) formula was stored in closed amber color glass vials at different conditions, that is, refrigerated condition $\left(5 \pm 2{ }^{\circ} \mathrm{C}\right)$, room temperature $\left(23 \pm 2{ }^{\circ} \mathrm{C}\right)$ and stress condition, i.e. high temperature $\left(40 \pm 2{ }^{\circ} \mathrm{C}, 75 \% \mathrm{RH}\right)$. The sample was withdrawn at a predetermined time interval, that is, 6 and $12 \mathrm{w}$ and the clarity, $\mathrm{pH}$ and drug content were analysed.

\section{Statistical analysis}

The experimental data were analyzed using Origin software (version 8.0, OriginLab), one-way Analysis of Variance (ANOVA) test was applied and a p-value of less than 0.05 was considered as the required level of significance $(n=3)$.

\section{RESULTS AND DISCUSSION}

\section{Clarity and $\mathbf{p H}$}

Non-transparent formulations might blur the vision and are not tolerable by the patient. So clarity is an extremely desirable characteristic in ophthalmic formulations. All prepared ciprofloxacin $\mathrm{HCl}$ in-situ gel formulas were clear and transparent. The $\mathrm{pH}$ values of all formulas were found to be between 4.57 and 4.72 as shown in table 2. These values comply with pharmacopeial requirements [19].

\section{Drug content}

The drug content of all formulas was in the range of (94.01$102.59 \%$ ) as shown in table 2 which is in compliant with the requirement of the assay $(90-110 \%)$ [19].

\section{Fourier transform infrared spectroscopy (FTIR)}

FTIR results showed no detected chemical interaction between ciprofloxacin $\mathrm{HCl}$ and P407. Fig. 2 shows that there were no extra peaks appearing and no noticed shift in peaks when comparing the IR chart of ciprofloxacin alone (fig. 2, a), the IR chart of poloxamer alone (fig. 2, b) with the IR chart of the physical mixture of ciprofloxacin $\mathrm{HCl}$ and P407 (fig. 2, c).
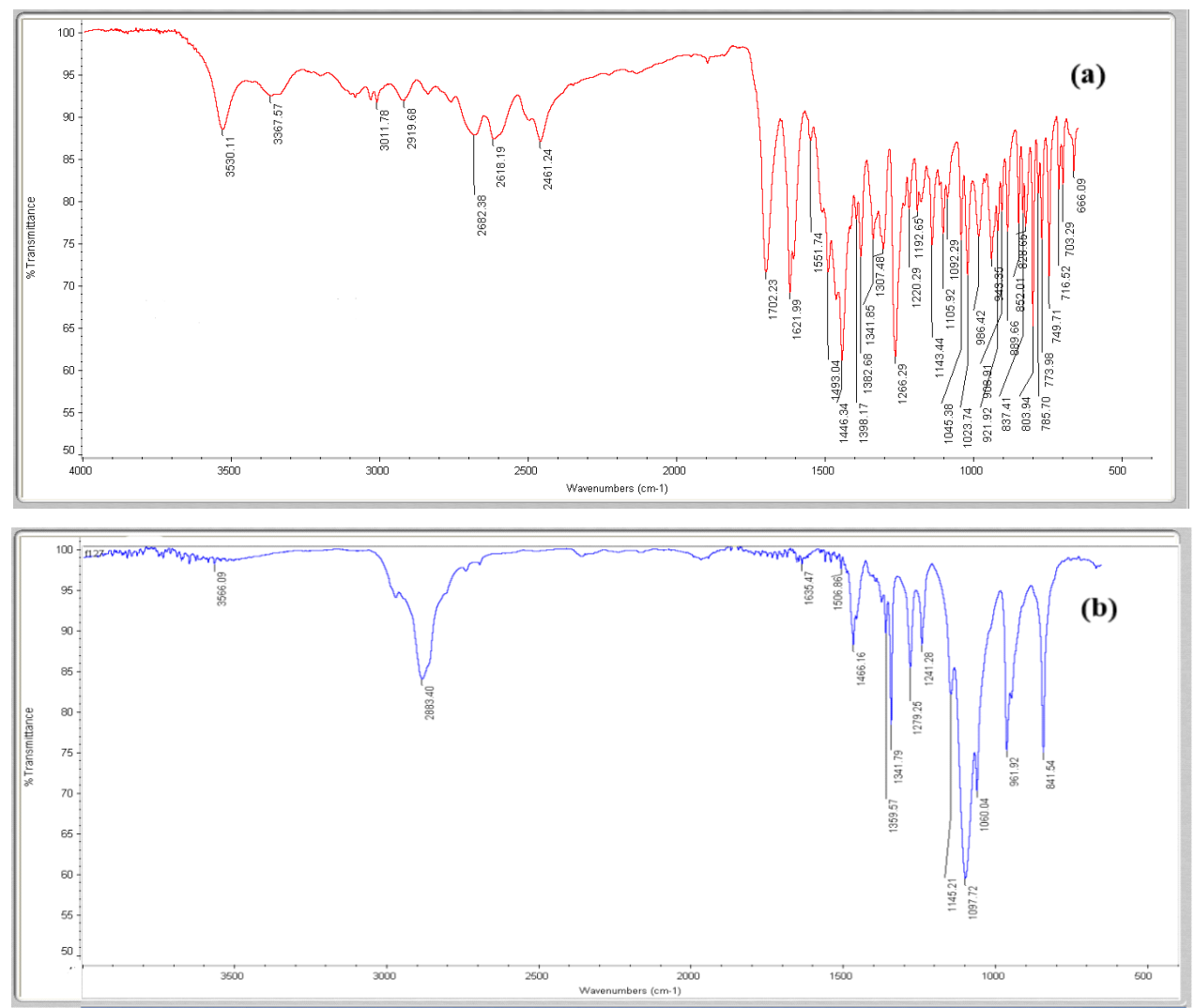


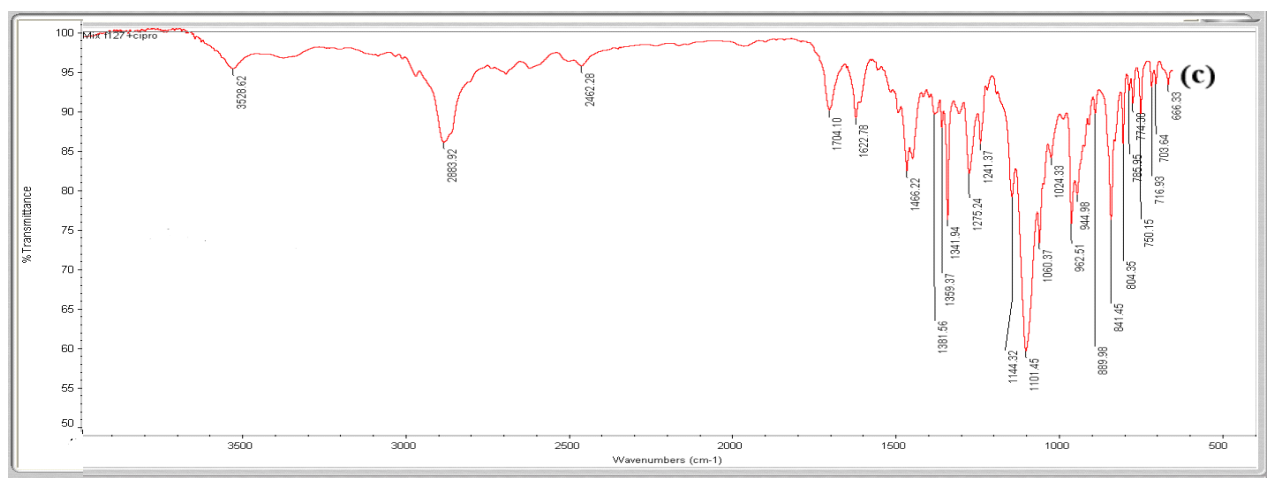

Fig. 2: FTIR spectra for ciprofloxacin hydrochloride (a), poloxamer 407 (P407) (b), and the physical mixture of ciprofloxacin HCl and poloxamer 407 (ciprofloxacin-P407PM) (c)

\section{Measurement of the gelation temperature}

The best in-situ gel ocular system should be liquid with low viscosity at room temperature (around $23^{\circ} \mathrm{C}$ ) to facilitate administration into the eye as drops. However, at physiological temperature $\left(37^{\circ} \mathrm{C}\right)$ it should undergo a phase change into a gel in the ocular cul-de-sac to prolong drug release. The formulas produced using 15\% P407 failed to form a gel at physiological temperature. Using higher concentrations of P407; 16.25\% (F4, F5, F6 and F11), 17.5\% (F7, F8, F9 and F12) and 20\% (F10) produced clear gel (table 2). Fig. 3 shows the viscosity changes in consequence with gel-forming when the temperature has been increased from 22 to $34^{\circ} \mathrm{C}$.

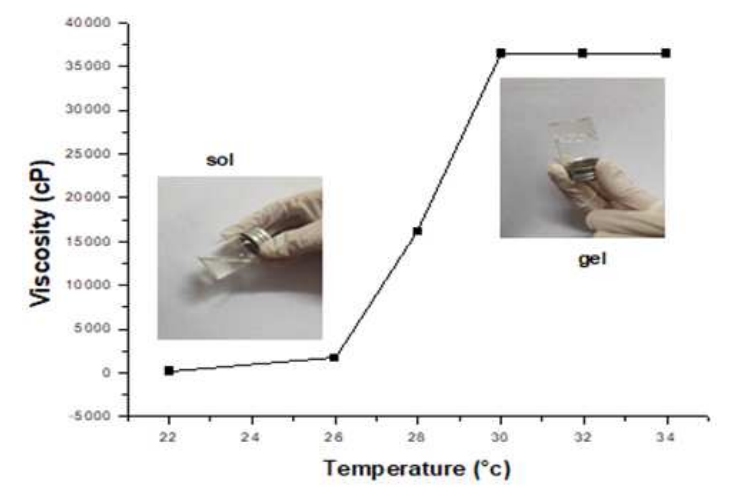

Fig. 3: Phase change or solution-gel transition at different temperatures. Values represent mean $\pm S D .(n=3)$

A relationship between gelation temperature and the concentration of P407 was noticed as demonstrated in (fig. 4); when poloxamer
407 concentration increases there was a significant reduction in gelation temperature $(* \mathrm{p}$ less than 0.05$)$. This is consistent with many works [20-23]. Park et al. found a linear relationship between P407 concentration and sol-gel transition temperature over a concentration range of $10-25 \%$; the transition temperature is linearly reduced when P407 concentration has been increased [24].

Having more effective concentration of $\mathrm{P} 407$ retain a higher number of micelles to be contained in formulas. As a result, they would need lesser energy to promote sol-gel transition and this lead to achieve a transition at a lower temperature than formulas containing lower P407 content.

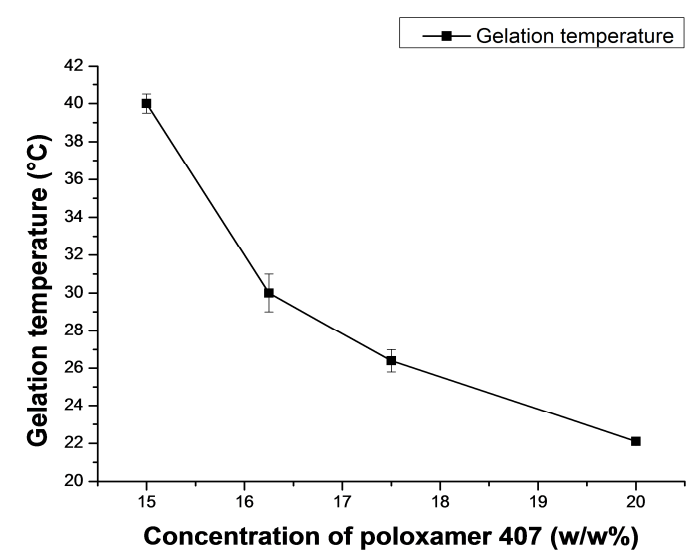

Fig. 4: Gelation temperature of different concentrations of poloxamer 407 , values represent mean \pm SD. $(n=3)$

Table 2: Evaluation parameters of various formulas of ocular in-situ gels of ciprofloxacin hydrochloride

\begin{tabular}{|c|c|c|c|c|c|c|}
\hline Formulas & pH & clarity & Transparency & Temp. Sol/gel ${ }^{\circ} \mathrm{C}$ & $\begin{array}{l}\text { Gelling } \\
\text { capacity }\end{array}$ & $\begin{array}{l}\text { Drug content } \\
\%\end{array}$ \\
\hline F1 & $4.64 \pm 0.01$ & Clear & transparent & No gel up to 40 & + & $100.69 \pm 0.08$ \\
\hline F2 & $4.64 \pm 0.00$ & Clear & transparent & No gel up to 40 & + & $94.01 \pm 0.02$ \\
\hline F3 & $4.63 \pm 0.01$ & Clear & transparent & No gel up to 40 & + & $96.91 \pm 0.11$ \\
\hline F4 & $4.61 \pm 0.00$ & Clear & transparent & $31.5 \pm 0.3$ & ++ & $95.5 \pm 0.06$ \\
\hline F5 & $4.66 \pm 0.00$ & Clear & transparent & $31.1 \pm 0.1$ & +++ & $96.06 \pm 0.06$ \\
\hline F6 & $4.57 \pm 0.00$ & Clear & transparent & $30 \pm 0.01$ & +++ & $98.35 \pm 0.11$ \\
\hline F7 & $4.72 \pm 0.00$ & Clear & transparent & $26.8 \pm 0.2$ & +++ & $94.2 \pm 0.03$ \\
\hline F8 & $4.72 \pm 0.03$ & Clear & transparent & $26.3 \pm 0.1$ & +++ & $99.53 \pm 0.01$ \\
\hline F9 & $4.72 \pm 0.01$ & Clear & transparent & $25.9 \pm 0.3$ & +++ & $102.59 \pm 0.02$ \\
\hline F10 & $4.72 \pm 0.00$ & Clear & transparent & $22 \pm 0.1$ & ++++ & $99.3 \pm 0.22$ \\
\hline F11 & $4.63 \pm 0.00$ & Clear & transparent & $31.6 \pm 0.05$ & ++ & $101.7 \pm 0.04$ \\
\hline F12 & $4.65 \pm 0.01$ & Clear & transparent & $26.8 \pm 0.1$ & +++ & $97.4 \pm 0.03$ \\
\hline
\end{tabular}

Note: + , Viscous solution formed after a few minutes, dissolves rapidly; ++ , Immediate gelation and remains for few hours (moderate gelling capacity); +++, Immediate gelation and remains for extended period (high gelling capacity);++++,viscous gel, $\mathrm{n}=3$; Data are expressed as mean \pm SD. 


\section{Gelling capacity}

The gelling capacity of all formulas is presented in table 2 . Formulas F1 to F3, which were prepared with low concentrations of poloxamer (15\%), formed viscous solution after a few minutes which dissolves rapidly. However, formulas with higher concentrations of poloxamer (F4-F12) showed immediate gelation and remained as a gel for an extended period of time. The ideal formula should undergo a rapid gelation in the ocular cul-de-sac and the gel formed in-situ should reserve its integrity without eroding or dissolving for an extended period of time [25]. F5-F12 showed high gelling capacity except for F11, which shows moderate gelling capacity. This might be attributed to that F11 contains no HPMC.

\section{Rheological study}

Fig. 5 presents the effect of different concentrations of poloxamer on the viscosity of the system. As the P407 concentration increases, there is an increase in viscosity. This may be due to the fact that poloxamer is nonionic polyoxyethylene-polyoxypropylene-polyoxyethylene triblock copolymer molecules that aggregate into micelles at $34{ }^{\circ} \mathrm{C}$ due to the dehydration of the polymer blocks with temperature. The gel formation is a result of micellar expansions and packing, and the gel is more entangled at greater poloxamer concentrations. As a result of these entanglements, micelles cannot separate easily from each other, consequently, a gel containing high concentrations of poloxamer would have rigidity and high viscosity $[26,27]$. Similar observations were found by Mai et al. and W. Lihong et al. who observed that the viscosity of poloxamer in-situ gel increased by increasing the concentration of P407 [26, 28]. Metoprolol tartrate ophthalmic gels, which prepared by Abou el Ela et al. found that the gels containing 30\% P407 showed a higher viscosity than that of $25 \%$ [27].

It is important to detect whether the rheological behavior of the formula is Newtonian or non-Newtonian. The formulas encoded F1 to F6, F11 and F12 exhibit Newtonian behavior at $22{ }^{\circ} \mathrm{C}$ (i.e. nonphysiological condition) while at $36^{\circ} \mathrm{C}$ (i.e. physiological condition),
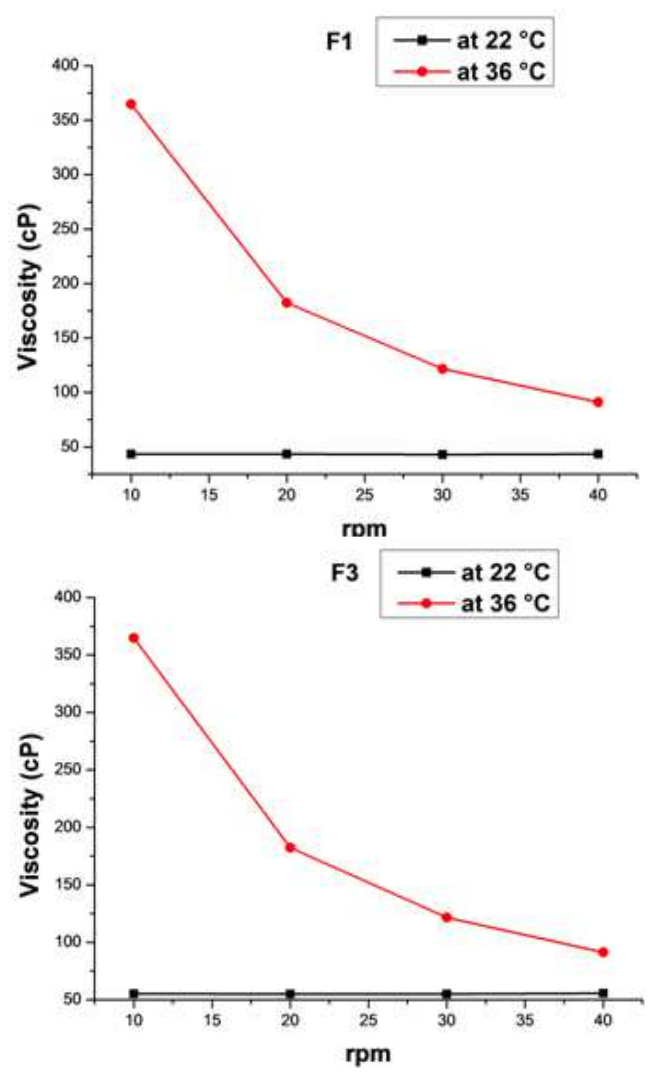

they show non-Newtonian shear thinning effect as shown in fig. 6. As the ocular shear rate, ranging from $0.03 \mathrm{~s}^{-1}$ during inter-blinking periods to $4250-28,500 \mathrm{~s}^{-1}$ during blinking, the formula has pseudoplastic shear thinning property under high stress is often favored $[29,30]$. Accordingly, high viscosity under the low shear rate and low viscosity under the high shear rate can increase contact time and decrease eye irritation after instillation.

It is worthy to note that F8, F9, and F10 showed non-Newtonian behavior at both physiological and non-physiological conditions. This might be due to the relatively high concentration of P407 used in these formulas. Varshosaz et al. found that increasing in the concentration of P407 up to $30 \%$ lead to gels display a nonNewtonian behavior at both non-physiological and physiological environments [31].

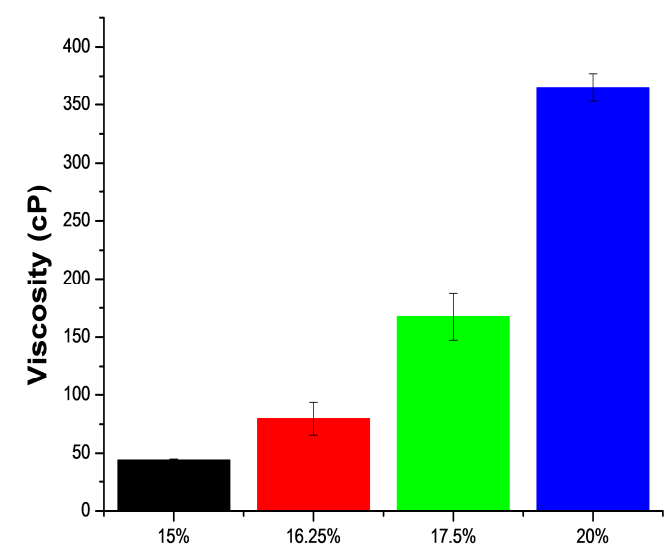

Fig. 5: Viscosity of in-situ gel at different poloxamer concentration. $n=3$; Data are expressed as mean $\pm S D$
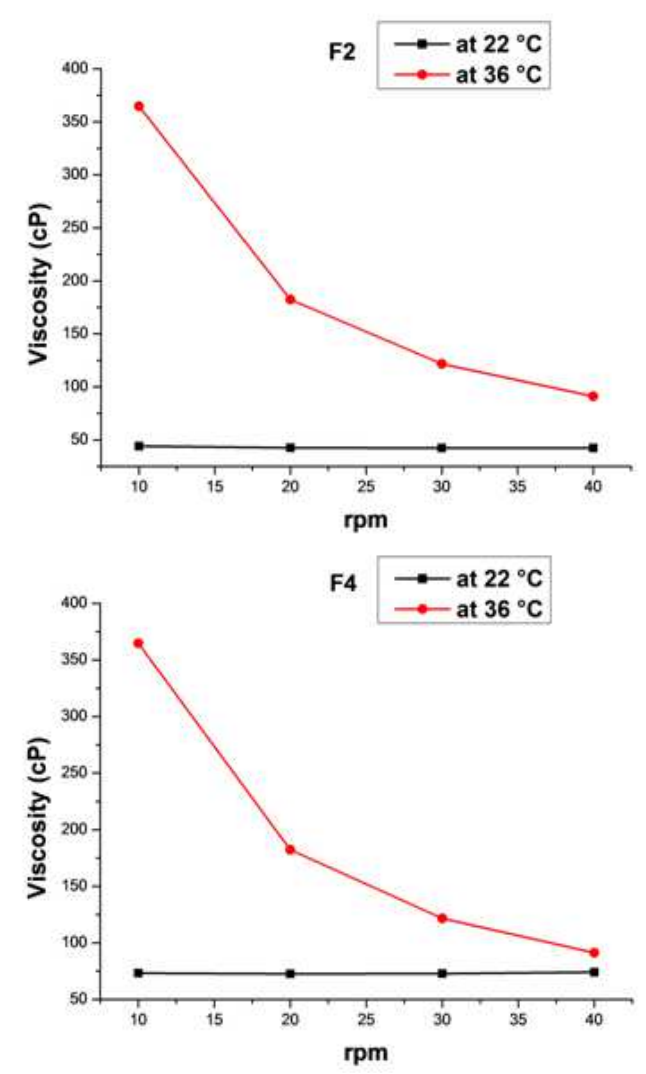

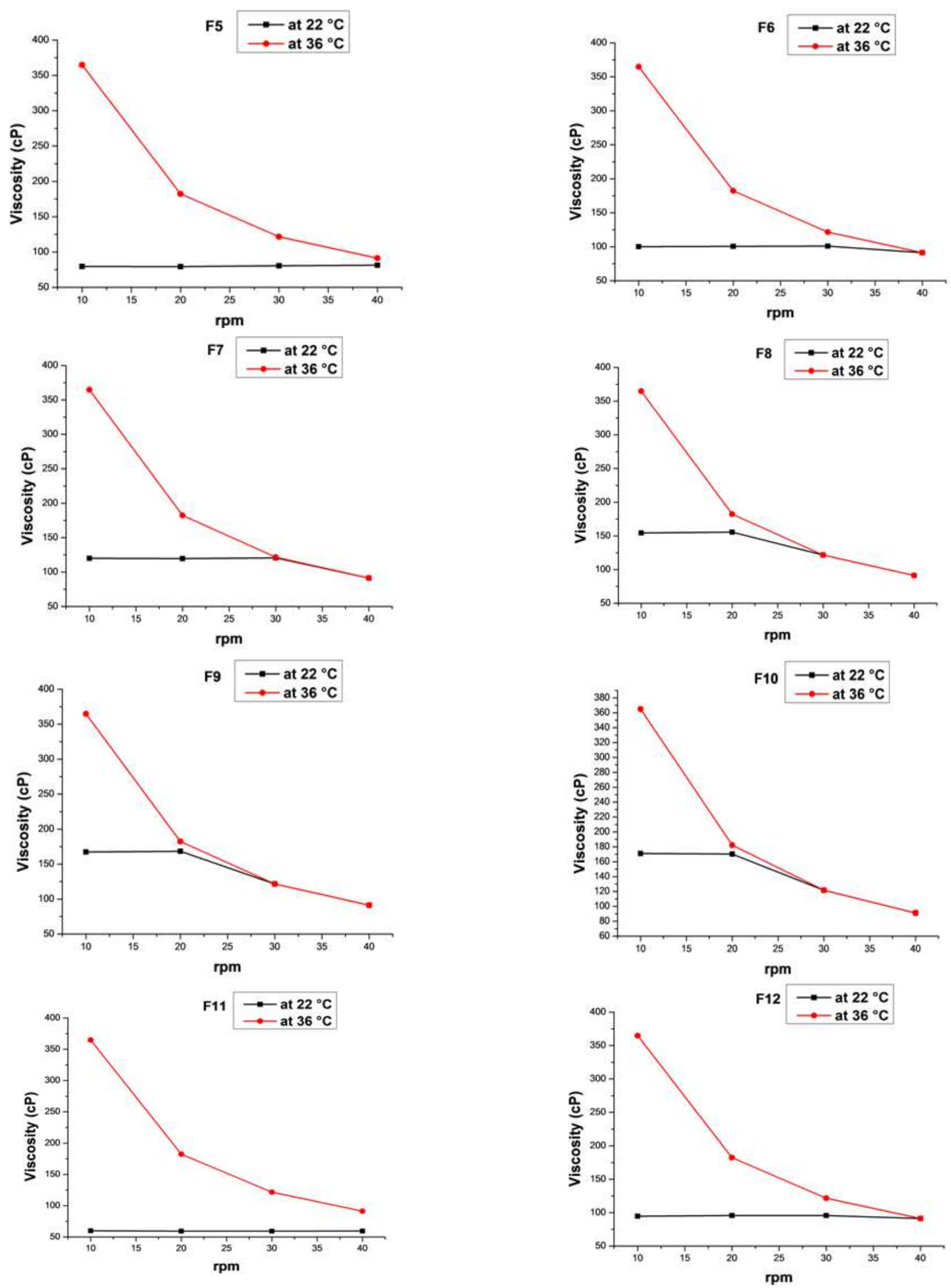

Fig. 6: Viscosity versus shear rate of all formulas at $22^{\circ} \mathrm{C}$ and $36^{\circ} \mathrm{C} .(\mathrm{n}=3$, Data presented as mean)

In addition, fig. 7 shows that the increment in temperature leads to increase in viscosity and the temperature at which viscosity displays a significant increment is noted as the gelation temperature [32]. When the temperature is increased, hydration of the polymer is gradually debilitated and polymer-polymer associations become more obvious, thus resulting in the formation of a gel with an increase in viscosity [33]

\section{Effect of HPMC on the viscosity}

The viscosity modifier, HPMC, was used in different concentrations. Fig. 7 shows the effect of HPMC on viscosity. It is clear that increasing HPMC concentration leads to increase in viscosity of the formula. This may be due to the fact that block copolymer P407 thermo sensitive gels are supposed to be formed by hydrogen 
bonding in aqueous systems, caused by the attraction of the poloxamer ether oxygen atom with protons of water. The number of hydrogen bonds is estimated to increase by adding compounds with hydroxyl groups such as cellulose derivative (HPMC), thus resulting in an increase in the measured viscosity of the prepared formulas [26].
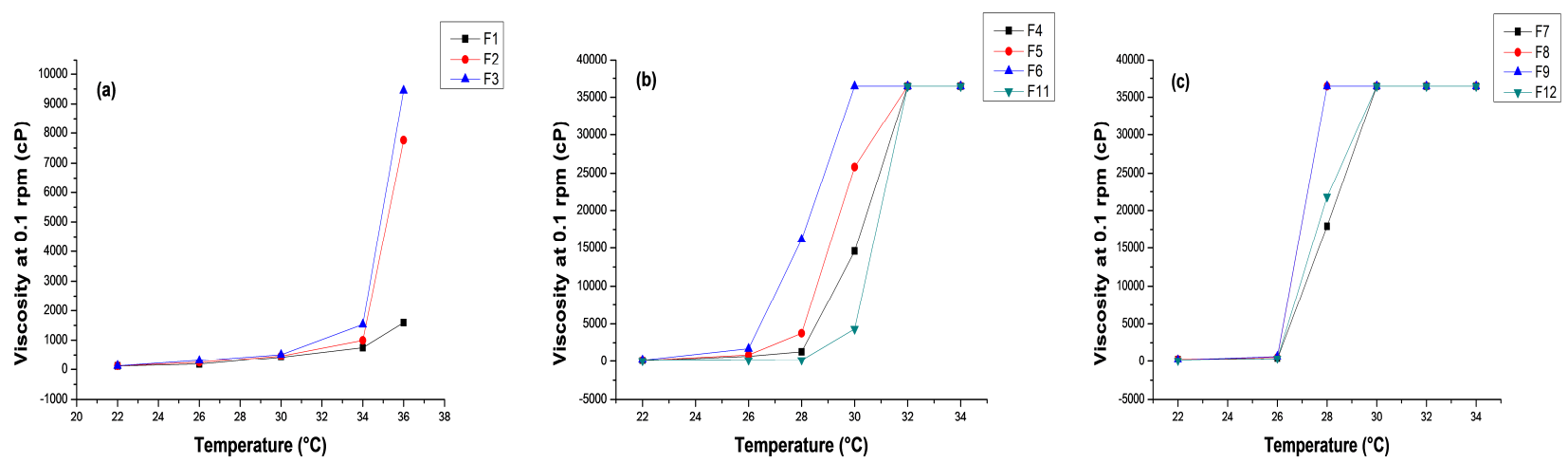

Fig. 7: Viscosities at $0.1 \mathrm{rpm}$ of different poloxamer formulas at different temperatures. (a) $15 \%$ poloxamer formulas with $0.2,0.4$ and $0.6 \%$ of HPMC for F1, F2 and F3 respectively. (b) 16.25\% poloxamer formulas with $0,0.2,0.4$ and $0.6 \%$ of HPMC for F11, F4, F5 and F6 respectively. (c) $17.5 \%$ poloxamer formulas with $0,0.2,0.4$ and $0.6 \%$ of HPMC for F12, F7, F8 and F9 respectively. (n=3, Data presented as mean)

\section{In vitro drug release study}

The cumulative percentage of ciprofloxacin $\mathrm{HCl}$ release profiles for various in-situ gel formulas in STF is shown in fig. 8a. As the formulas prepared with $16.25 \%$ expressed good gelling capacity, they were subjected to a further test of in vitro release. All tested formulas exhibited prolonged release.

In case of $\mathrm{F} 4$ and $\mathrm{F} 11$ about $50 \%$ of the ciprofloxacin $\mathrm{HCl}$ was released into the medium within $30 \mathrm{~min}$, and then about $80 \%$ was released in $8 \mathrm{~h}$.

F5 and F6 showed about $80 \%$ and $83 \%$ release in $8 \mathrm{~h}$, respectively, while about $40 \%$ of ciprofloxacin $\mathrm{HCl}$ was released within $30 \mathrm{~min}$ for both of them.

Remarkably, F12 shows longer release pattern, where about 38\% of the ciprofloxacin $\mathrm{HCl}$ was released into the medium within $30 \mathrm{~min}$ and then about $77 \%$ in $8 \mathrm{~h}$. F12 was prepared with $17.25 \%$ poloxamer and this might be the reason for the slow release of this formula. From the results, it is possible to conclude that when the concentration of P407 increased from 16.25 to $17.5 \%$, the amount of the drug released was reduced. These results show that the structure of the gel works as an increasingly resistant barrier to drug release as the concentration of P407 increased. The mechanism for such increased resistance may possibly be due to a decrease in the number and dimensions of water channels and an increase in the number and size of micelles inside the gel structure. The shorter intermicellar distance result in larger numbers of cross-links between neighboring micelles leading to greater viscosity and a lower rate of drug release [34]. This assumption may be supported by the rheology study which shows direct proportionality between P407 concentration and viscosity.

Fig. $8 \mathrm{~b}$ shows that in comparison to the marketed eye drop solution, where almost all the ciprofloxacin $\mathrm{HCl}$ was released immediately within $10 \mathrm{~min}$, the selected formula (F6) manifested prolonged release. This result indicates that in-situ gel formulation has prolonged-release effect in vitro in comparison to the low bioavailable, marketed formulation of ciprofloxacin eye drop.

\section{Ocular irritation test}

The result of eye irritation test of F6 in rabbits, studied by using modified Draize test, showed that there was no conjunctival redness or edema, no corneal opacity, no secretion and no iris involvement. Applying criteria adapted from Asasutjarit et al. for ocular irritation evaluation [35], showed that F6 did not irritate the rabbits' eyes since the total score of an eye irritation assessment was zero. Therefore, F6 can be considered safe for ophthalmic administration.
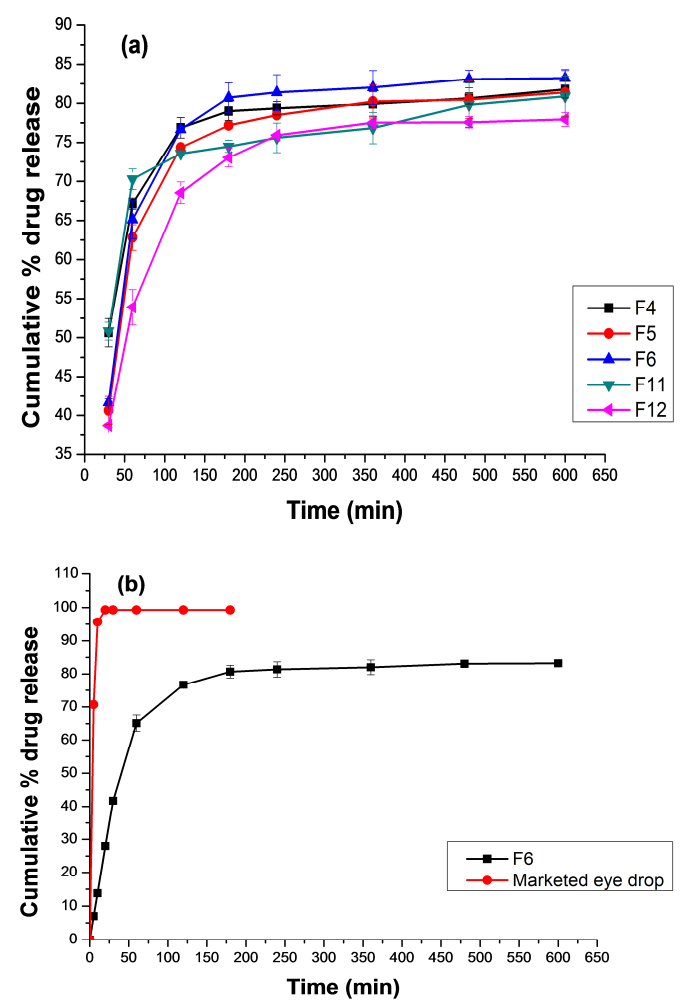

Fig. 8: Cumulative percentage drug release of different formulas. (a) Drug release from formulas prepared with $16.25 \%$ poloxamer in presence of different concentrations of HPMC (b) Drug release from the selected formula (F6) in comparison to the marketed eye drop. $n=3$; Data are expressed as mean $\pm S D$

\section{In vivo fluorescein tracking study}

Fluorescein was used in tracking the presence of gel in comparison to solution drop. As demonstrated in (fig. 9 a), in-situ gel, which is applied in the left eye, remained within the eye and slowly disappeared as the gel was cleared. While for the control right eye, a solution eye drop was applied (fig. 9 b), where a continuous drainage of fluorescein towards the inner canthus along the eyelid margin was seen. Thus, elimination of solution in rabbit right eye and gel in rabbit left eye was completed within $10 \mathrm{~min}$ and $60 \mathrm{~min}$, 
respectively. This result is even better than that obtained by Song $e t$ al. (2013) where the percorneal retention was improved from 10 min in case of the solution to $25 \mathrm{~min}$ in case of in-situ gel [36]. Improvement in percorneal retention time will lead to improved local bioavailability, reduce dose frequency and concentration and may improve patient compliance.

\section{Sterility test}

The formula F6 passed the test for sterility since there was no appearance of turbidity and no sign of microbial growth when incubated for $14 \mathrm{~d}$ at $30-35^{\circ} \mathrm{C}$ in case of fluid thioglycolate medium and at $20-25^{\circ} \mathrm{C}$ in case of soybean-casein digest medium. (a)

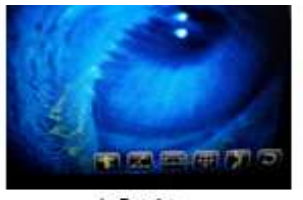

$1=5 \mathrm{~min}$

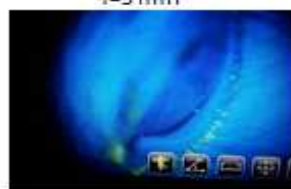

$\mathrm{IV}=30 \mathrm{~min}$

(b)

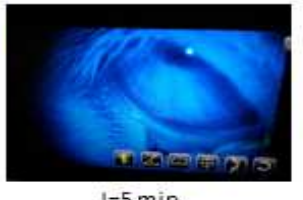

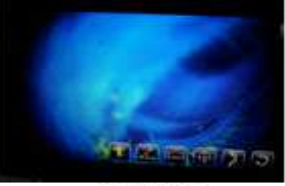

$\|=10 \mathrm{~min}$

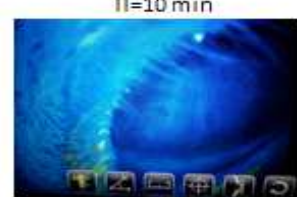

$\mathrm{V}=40 \mathrm{~min}$

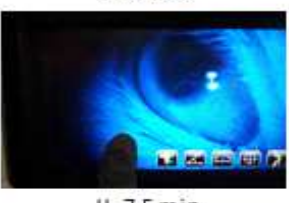

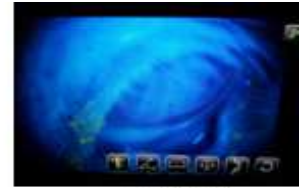

$\| I I=20 \mathrm{~min}$

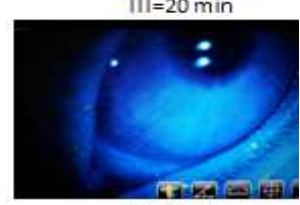

$\mathrm{V} l=60 \mathrm{~min}$

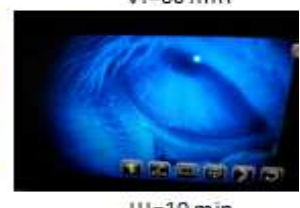

$I I I=10 \mathrm{~min}$

Fig. 9: In vivo elimination study, fluorescein sodium salt was dispersed in the preparations and visualized using an auto-refractometer.

One drop of each preparation was added into the lower conjunctival sac of rabbit, an auto-refractometer with blue light was used to monitor and photograph the disappearance of the fluorescein sodium salt. (a) Elimination time after the in-situ gel was dropped. (I-VI: 5,

10, 20, 30, 40, $60 \mathrm{~min}$ ). (b) Elimination time after the control marketed eye drop was dropped. (I-III: 5, 7.5, $10 \mathrm{~min}$ ). $\mathrm{n}=3$

\section{Stability test}

Formula 6 showed acceptable stability as shown in table 3, the drug content of formula 6 , when stored at $4{ }^{\circ} \mathrm{C}$ and at room temperature for $3 \mathrm{mo}$, showed no significant variations $\left({ }^{*} \mathrm{p}\right.$ less than 0.05$)$. In addition, the physical appearance of formula F6 when stored in a refrigerator and at room temperature did not change. However, F6 became gel after $3 \mathrm{mo}$ of storage at $40^{\circ} \mathrm{C}$. The pH of F6 when stored at $4{ }^{\circ} \mathrm{C}$ and at room temperature showed no significant variations while when stored at $40^{\circ} \mathrm{C}$ there was a significant variation $\left({ }^{*} \mathrm{p}\right.$ less than 0.05). This variation in the $\mathrm{pH}$ can be attributed to the dehydration of the gel formula.

Table 3: Stability of optimized formula F6 at different conditions

\begin{tabular}{llllllllll}
\hline & $\mathbf{0 ~ w}$ & & & $\mathbf{6 ~ w}$ & & & $\mathbf{1 2} \mathbf{w}$ & \\
\cline { 2 - 9 } & $\mathbf{p H}$ & Clarity & Drug content & $\mathbf{p H}$ & Clarity & Drug content & $\mathbf{p H}$ & Clarity & Drug content \\
\hline $4^{\circ} \mathrm{C}$ & $4.57 \pm 0.0$ & clear & $98.35 \pm 0.11$ & $4.6 \pm 0.01$ & clear & $98.2 \pm 0.0$ & $4.62 \pm 0.1$ & Clear sol & $98.01 \pm 0.1$ \\
$25^{\circ} \mathrm{C}$ & $4.57 \pm 0.0$ & clear & $98.35 \pm 0.11$ & $4.63 \pm 0.0$ & clear & $98.7 \pm 0.2$ & $4.63 \pm 0.0$ & Clear sol & $97.9 \pm 0.06$ \\
$40^{\circ} \mathrm{C}$ & $4.57 \pm 0.0$ & clear & $98.35 \pm 0.11$ & $5.11 \pm 0.01$ & clear & $103.1 \pm 0.0$ & $5.41 \pm 0.0$ & Gel formed & Not measured \\
\hline
\end{tabular}

$\mathrm{n}=3$; Data are expressed as mean \pm SD.

\section{CONCLUSION}

Ciprofloxacin, a broad-spectrum antibacterial agent used in the treatment of ocular infections, was successfully formulated as thermo-sensitive ophthalmic in-situ gel using poloxamer 407 as a gelling agent in combination with HPMC as a viscosity-enhancing agent. Upon instillation into the eye as drops, the formula undergoes gelation in the cul-de-sac. The formed gel expressed prolonged in vitro drug release over $8 \mathrm{~h}$ period. The optimized formula (F6) represents a promising alternative to the conventional eye drops due to its ability to enhance availability through its prolonged drug release and longer precorneal residence time. In addition, the ease of administration and the expected reduced frequency of administration will result in better patient compliance.

\section{ACKNOWLEDGEMENT}

Great thanks is expressed to Pioneer Company for the pharmaceutical industry (Sulaymania, Iraq) for helping in conducting part of this study in their lab.

\section{AUTHORS CONTRIBUTIONS}

All the author have contributed equally

\section{CONFLICTS OF INTERESTS}

\section{Declared none}

\section{REFERENCES}

1. Ludwig A. The use of mucoadhesive polymers in ocular drug delivery. Adv Drug Deliv Rev 2005;57:1595-639.

2. Almeida H, Amaral MH, Lobão P, Lobo JMS. In situ gelling systems: a strategy to improve the bioavailability of ophthalmic pharmaceutical formulations. Drug Discovery Today 2014;19:400-12.

3. Shah JN. Formulation, optimization and characterization of novel controlled ophthalmic drug delivery systems of some fluoroquinolones. Ganpat University; 2011.

4. Horvat G, Gyarmati B, Berko S, Szabo-Revesz P, Szilagyi BA, Szilágyi A, et al. Thiolated poly(aspartic acid) as potential in situ gelling, ocular mucoadhesive drug delivery system. Eur J Pharm Sci 2015;67:1-11. 
5. Morsi N, Ibrahim M, Refai H, El Sorogy H. Nanoemulsion-based electrolyte triggered in situ gel for ocular delivery of acetazolamide. Eur J Pharm Sci 2017;104:302-14.

6. Almeida $\mathrm{H}$, Amaral $\mathrm{MH}$, Lobao $\mathrm{P}$ SLJ. Applications of poloxamers in ophthalmic pharmaceutical formulations: an overview. Expert Opin Drug Delivery 2013;10:1223-37.

7. Dumortier G, Grossiord JL, Agnely F, Chaumeil JC. A review of poloxamer 407 pharmaceutical and pharmacological characteristics. Pharm Res 2006;23:2709-28.

8. Abdelkader H, Mansour HF. Comparative studies for ciprofloxacin hydrochloride pre-formed gels and thermally triggered (in situ) gels: in vitro and in vivo appraisal using a bacterial keratitis model in rabbits. Pharm Dev Technol 2015;20:410-6.

9. BP British Pharmacopoeia. Stationary Office 2009; 2010. p. 5000.

10. Patel N, Thakkar V, Metalia V, Baldaniya L, Gandhi T, Gohel M. Formulation and development of ophthalmic in situ gel for the treatment ocular inflammation and infection using application of quality by design concept. Drug Dev Ind Pharm 2016;42:1406-23.

11. Shashank Nayak N, Sogali BS, Thakur RS. Formulation and evaluation of pH-triggered in situ ophthalmic gel of Moxifloxacin hydrochloride. Int J Pharm Pharm Sci 2012;4:452-9.

12. Destruel PL, Zeng N, Maury M, Mignet N, Boudy V. In vitro and in vivo evaluation of in situ gelling systems for sustained topical ophthalmic delivery: state of the art and beyond. Drug Discovery Today 2017;22:638-51.

13. Almeida H, Lobao P, Frigerio C, Fonseca J, Silva R, Quaresma P, et al. Development of mucoadhesive and thermosensitive eyedrops to improve the ophthalmic bioavailability of ibuprofen. J Drug Delivery Sci Technol 2016;35:69-80.

14. Rathore KS. In-situ gelling ophthalmic drug delivery system: an overview. Int J Pharm Pharm Sci 2010;2:30-4.

15. Lin HR, Sung KC. Carbopol/pluronic phase change solutions for ophthalmic drug delivery. J Controlled Release 2000;69:379-88.

16. Allah AKA, Hammid SNA. Preparation and evaluation of chloramphenicol as a thermosensitive ocular in-situ gel. Iraqi J Pharm Sci 2012;21:98-105.

17. El-Laithy HM, Nesseem DI, El-Adly AA, Shoukry M. Moxifloxacin-gelrite in situ ophthalmic gelling systems against photodynamic therapy for treatment of bacterial corneal inflammation. Arch Pharm Res 2011;34:1663-78.

18. Deshpande J, Shah PB, Bhandari A. Design and development of pH-monitered in situ gel of Lomefloxacin. J Pharm Sci Biosci Res 2013;3:10-5.

19. USP $32 \mathrm{NF}$ 27. 32nd ed. United States Pharmacopeial Convention; 2009.

20. MA Fathalla Z, Vangala A, Longman M, Khaled KA, Hussein AK, El-Garhy $\mathrm{OH}$, et al. Poloxamer-based thermoresponsive ketorolac tromethamine in situ gel preparations: design, characterisation, toxicity and transcorneal permeation studies. Eur J Pharm Biopharm 2017;114:119-34.
21. Galgatte UC, Chaudhari PD. Preformulation study of poloxamer 407 gels: effect of additives. Int J Pharm Pharm Sci 2014;6:130-3.

22. Cao F, Zhang X, Ping Q. New method for ophthalmic delivery of azithromycin by poloxamer/carbopol-based in situ gelling system. Drug Delivery 2010;17:500-7.

23. Sheshala R, Quah SY, Tan GC, Meka VS, Jnanendrappa N, Sahu PS. Investigation on a solution-to-gel characteristic of thermosensitive and mucoadhesive biopolymers for the development of moxifloxacin-loaded sustained release periodontal in situ gels. Drug Delivery Transl Res 2018;1:1-10.

24. Park E, Song K. Rheological properties of poloxamer 407 solutions and gels. Annu Trans Nord Rheol Soc 2011;19:1-5.

25. Wu H, Liu Z, Peng J, Li L, Li N, Li J, et al. Design and evaluation of baicalin-containing in situ pH-triggered gelling system for sustained ophthalmic drug delivery. Int J Pharm 2011;410:31-40.

26. Mansour M, Mansour S, Mortada ND, Abd ElHady SS. Ocular poloxamer-based ciprofloxacin hydrochloride in situ forming gels. Drug Dev Ind Pharm 2008;34:744-52.

27. Abou el Ela AESF, Khatib MM El. Formulation and evaluation of new long-acting metoprolol tartrate ophthalmic gels. Saudi Pharm J 2014;22:555-63.

28. Lihong W, Xin C, Yongxue G, Yiying B, Gang C. Thermoresponsive ophthalmic poloxamer/tween/carbopol in situ gels of a poorly water-soluble drug fluconazole: preparation and in vitro-in vivo evaluation. Drug Dev Ind Pharm 2014;40:1402-10.

29. Bachhav HD, Savkare A, Karmarkar R, Derle D. Development of poloxamer-based thermosensitive in situ ocular gel of betaxolol hydrochloride. Int J Pharm Pharm Sci 2015;7:4-8.

30. Paradkar MU, Parmar M. Formulation development and evaluation of natamycin niosomal in-situ gel for ophthalmic drug delivery. J Drug Delivery Sci Technol 2017;39:113-22.

31. Varshosaz J, Tabbakhian M, Salmani Z. Designing of a thermosensitive chitosan/poloxamer in situ gel for ocular delivery of ciprofloxacin. Open Drug Delivery J 2008;2:61-70.

32. Dewan M, Sarkar G, Bhowmik M, Das B. Effect of gellan gum on the thermogelation property and drug release profile of Poloxamer 407 based ophthalmic formulation. Int J Biol Macromol 2017;102:258-65.

33. Bhowmik M, Kumari P, Sarkar G, Kanti M, Bhowmick B, Bhattacharjee D, et al. Effect of xanthan gum and guar gum on in situ gelling ophthalmic drug delivery system based on poloxamer-407. Int J Biol Macromol 2013;62:117-23.

34. Alexandridis P, Hatton TA. Poly(ethylene oxide)-poly(propylene oxide)-poly (ethylene oxide) block copolymer surfactants in aqueous solutions and at interfaces: thermodynamics, structure, dynamics, and modeling. Colloids Surfaces 1995;7757:1-46.

35. Asasutjarit R, Thanasanchokpibull S, Fuongfuchat A, Veeranondha S. Optimization and evaluation of thermoresponsive diclofenac sodium ophthalmic in situ gels. Int J Pharm 2011;411:128-35.

36. Song J, Bi H, Xie X, Guo J, Wang X, Liu D. Preparation and evaluation of sinomenine hydrochloride in situ gel for uveitis treatment. Int Immunopharmacol 2013;17:99-107. 